\title{
Electron Paramagnetic Resonance Study of Chromosilicalite
}

\author{
O. Nakamura \\ Instituto de Fisica, Universidade Federal da Bahia, 40210, Salvador, Bahia, Brazil
}

J. Silvio T. Mambrim, H. O. Pastore and E. J. S. Vichi

Instituto de Quimica, Universidade Estadual de Campinas, Caixa Postal 6154, 13081, Campinas,

São Paulo, Brazil

F. G. Gandra, E. C. da Silva and H. Vargas*

Instituto de Fisica, Universidade Estadual de Campinas, Caixa Postal 6165, 13081, Campinas, São Paulo, Brazil

\author{
J. Pelzl \\ Institut für Experimentalphysik VI, Ruhr Universität Bochum, P.O. Box 102148, D-4630 Bochum, \\ Federal Republic of Germany
}

\begin{abstract}
An electron paramagnetic resonance (EPR) study of crystalline chromosilicalite has been presented with the purpose of characterization of the distribution of $\mathrm{Cr}^{3+}$ sites in the solid. In addition, a deconvolution process is described whereby the EPR parameters can be analysed.
\end{abstract}

The catalytic importance of chromium has prompted numerous attempts to introduce chromium cations in zeolites. For example, Naccache and Ben Taarit, ${ }^{1}$ Atanasova et $a .^{2}{ }^{2}$ and Pearce et al. ${ }^{3}$ have investigated the $\mathrm{Cr}$ species mainly in $\mathrm{A}, \mathrm{X}$, and $\mathrm{Y}$ zeolites, whereas Hemidy et al. $^{4}$ and Kucherov and Slinkin ${ }^{5}$ reported results for the $\mathrm{Cr}$-mordenite system. In a recent communication ${ }^{6}$ we reported the incorporation of $\mathrm{Cr}^{3+}$ in the structural positions of ZSM-5 type zeolites, and recently we have demonstrated the incorporation of oxygen in this zeolite with oxidation of anchored $\mathrm{Cr}^{3+}$ to noninteracting $\mathrm{Cr}^{6+}$ species. $^{7}$

Here an EPR study of crystalline chromosilicalite is presented with the purpose of further characterizing the distributon of each $\mathrm{Cr}^{3+}$ site in the solid. Such characterization is a necessary prelude to establishing the chromosilicalite activity as a catalyst in the production of alkenes. In addition a deconvolution process is described whereby the EPR parameters could be analysed. This method proved to be quite successful, and the results provide evidence that $\mathrm{Cr}^{3+}$ ions are at least in two substitutional-like or highly distorted sites and in another one outside the silicon framework.

\section{Experimental}

\section{Material and Methods}

Synthesis

A mixture of sodium silicate (Riedel de-Haën: $63 \% \mathrm{SiO}_{2}$, $16 \% \mathrm{Na}_{2} \mathrm{O}$ and $18 \%$ water), ammonium fluoride, chromium nitrate 9-hydrate and water was prepared in the ratio $0.1: 0.05: 0.25: 6.0$, for the synthesis of a sample with $\mathrm{SiO}_{2}: \mathrm{Cr}_{2} \mathrm{O}_{3}$ ratio of $100: 1$ in the initial mixture. The $\mathrm{pH}$ was adjusted to $6.5-7.0$ with $48 \mathrm{vol} \% \mathrm{HF}$. After $2 \mathrm{~h}$ stirring, tetrapropylammonium bromide was added in $40 \mathrm{~cm}^{3}$ aqueous solution. The suspension was left to age for $24 \mathrm{~h}$, at 348- $353 \mathrm{~K}$, under ambient pressure. The hydrothermal treatment was performed at $443-448 \mathrm{~K}$ for 15 days. The crystallized chromosilicalite was then filtered, washed until free of $\mathrm{F}^{-}$and at neutral $\mathrm{pH}$ and air dried. The samples were kept in a desiccator over saturated $\mathrm{CaCl}_{2}$ solution before use. Elemental analysis showed the following composition for the assynthesized material (in \%): $\mathrm{SiO}_{2}, 81.60 ; \mathrm{Al}_{2} \mathrm{O}_{3}, 1.00$; $\mathrm{Cr}_{2} \mathrm{O}_{3}, 0.90 ; \mathrm{Fe}_{2} \mathrm{O}_{3}, 0.02 ; \quad \mathrm{Na}_{2} \mathrm{O}, 2.41$. The ratio $\mathrm{SiO}_{2}: \mathrm{Cr}_{2} \mathrm{O}_{3}$ is $743 .{ }^{11}$ The material is a highly crystalline
ZSM-5 molecular sieve, as shown by the ratio of 550 to 450 $\mathrm{cm}^{-1}$ absorbances of 0.75 for an as-synthesized sample and of 0.81 for a calcined sample.

\section{$E P R$}

EPR spectra were obtained using a Varian E-12 spectrometer operating at $9.5 \mathrm{GHz}$ (X-band). The $g$-values of the samples were obtained with reference to a standard mark: DPPH, $g$ value $=2.0036$. The spectra were recorded on samples at room $(300 \mathrm{~K})$ and liquid nitrogen $(77 \mathrm{~K})$ temperature.

\section{Computational Fittings}

The experimental data were fitted to theoretical expressions using an IBM 3090 computer. The expressions used were composed of the superposition of symmetrical line-shapes with powder pattern line-shapes. ${ }^{8}$ The routines used for the fittings are those from Bevington. ${ }^{9}$

\section{Results and Discussion}

Fig. 1 shows the spectra of the silicalite material (without chromium) and the effect of its calcination. The EPR spectra prior to calcination, at 300 and $77 \mathrm{~K}$ show a broad peak centred at $g \approx 2$. Superimposed on it there is a sharp peak at $g=1.97$. Also, there is another peak at $g=4.27$ due to $\mathrm{Fe}^{3+}$,

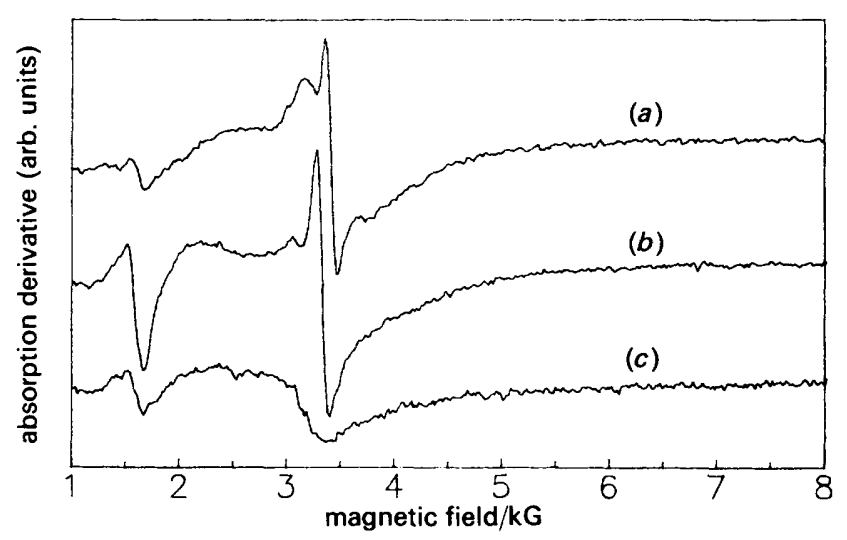

Fig. 1 EPR spectra of a silicalite sample $(a)$ as prepared, at room temperature, (b) at liquid nitrogen temperature, $(c)$ calcined for $24 \mathrm{~h}$ at $843 \mathrm{~K}$, at room temperature 


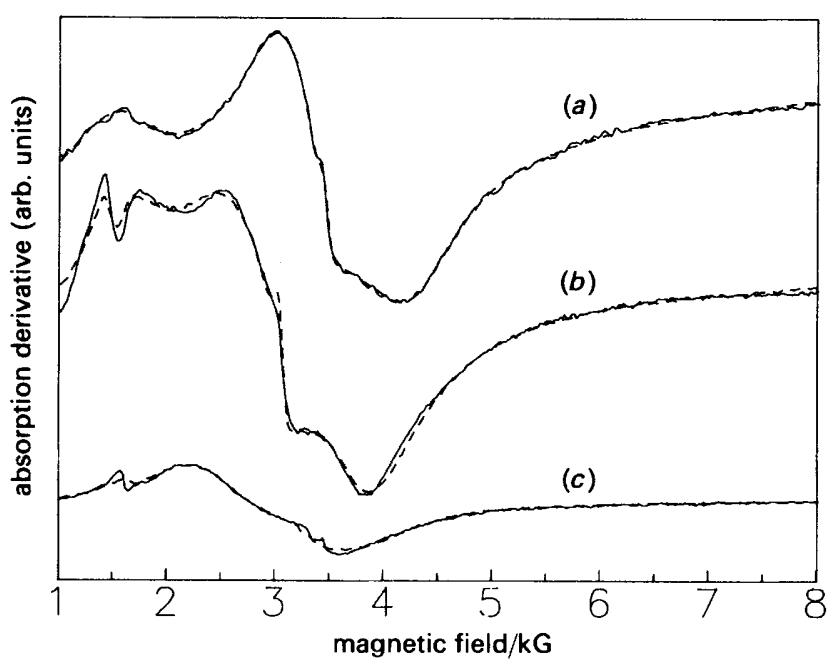

Fig. 2 EPR spectra of crystalline chromosilicalite $(a)$ at room temperature, $(b)$ at liquid nitrogen temperature, $(c)$ calcined for $24 \mathrm{~h}$ under oxygen flow. The measurements were carried out at room temperature

an impurity that comes from the silicon source. From the sample calcined at $843 \mathrm{~K}$ it can be seen that the broad and the sharp peak signals decreased drastically in intensity. The sharp peak at $g=1.97$ is due to free radicals formed from organic compounds used as template in the material preparation. These signals described above will serve as background for our EPR chromosilicalite spectra in an effort to elucidate the distribution of $\mathrm{Cr}^{3+}$ in the different sites in our zeolite.

Fig. 2 shows the spectra at (a) 300 and $(b) 77 \mathrm{~K}$ of a highly crystalline zeolitic chromosilicate containing $\mathrm{Cr}^{3+}$ both in framework and non-framework positions. The spectra show a distribution of resonances which includes high $g$-values. To interpret the complex spectra shown in Fig. 2, a deconvolution was made considering the following contributions: one symmetrical line centred on $g \approx 2.0$, the background spectrum and two powder pattern line-shapes. This is shown in Fig. 3. The dashed lines in Fig. 2 correspond to the best fit to the experimental data using the resonance field, linewidth and amplitude as adjustable parameters. The results obtained are presented in Table 1 . We assume that the two powder spectra resonances are due to different sites (A and B) in silicon framework positions. Both sites suffer the action of the crystalline field with different cubic symmetry distortions. The symmetrical line $(C)$ centred at $g=2.0$, has been attributed by several authors ${ }^{3}$ to the exchange cation $\left[\mathrm{Cr}\left(\mathrm{H}_{2} \mathrm{O}\right)_{6}\right]^{3+}$ and is outside the silicon framework. As expected the line centred at $g \approx 2.0$, corresponding to site $\mathrm{C}$, shows an increase in linewidth as the temperature decreases. There is a clear indication of interaction between $\mathrm{Cr}^{3+}$ ions for this site out of the silicon framework. Concerning the substitutional sites (A and B) we can see that the linewidth is

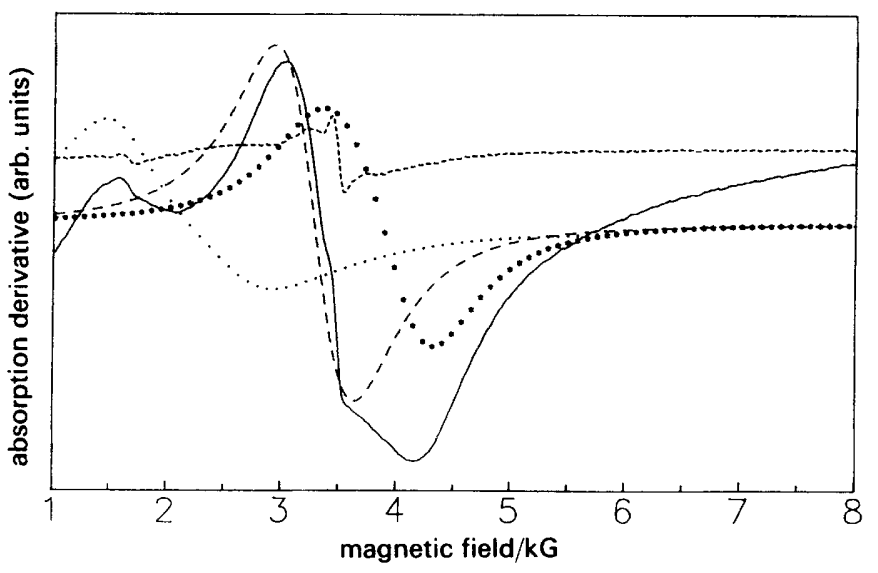

Fig. 3 Deconvolution of the experimental EPR curve of crystalline chromosilicalite at room temperature. (-- ), Background silicalite; $(--), g=2$, line $\mathrm{C} ;(\cdots)$, powder spectrum $\mathrm{A} ;(\bullet \bullet \bullet)$, powder spectrum $B ;(-)$, total spectrum

basically constant with temperature considering the complexity of the fitting procedures. This means a lower interaction of the $\mathrm{Cr}^{3+}$ ions.

We investigated the changes of EPR spectrum upon calcination of the sample for $24 \mathrm{~h}$ at $843 \mathrm{~K}$ under oxygen flow. The spectrum is shown in Fig. 2(c). The dashed line corresponds to the fitting of the experimental data. A comparison between Fig. 2(c) and (a) shows a considerable decrease in intensity of the calcined sample spectrum. This can be explained by a decrease in the number of cationic or charge-balancing sites, possibly as a consequence of oxidation of $\mathrm{Cr}^{3+}$ to $\mathrm{Cr}^{6+}$. The intensity of substitutional sites $\mathrm{A}$ and $\mathrm{B}$ also decreases due to ruptures in the zeolite framework resulting from calcination at $843 \mathrm{~K}$. The decrease in intensity of the narrow line centred at $g=1.97$ is also observed. This line, as discussed previously, is related to free radicals from organic compounds created during the sample preparation. This line has been noted by previous authors ${ }^{10}$ and was thought to arise from a small amount of $\mathrm{Cr}^{5+}$. However, we have observed a decrease in the intensity of this line for both the silicalite material (without chromium) and the chromosilicalite upon calcination. Hence, in the present case this decrease is due principally to the annealing and calcination of the free radicals.

The photoacoustic spectrum of the chromosilicalite ${ }^{7}$ is characterized by two bands centred at 440 and $640 \mathrm{~nm}$, respectively, corresponding to ligand field bands of $\mathrm{Cr}^{3+}$. For the level assignment the band centred at $440 \mathrm{~nm}$ is interpreted as the ${ }^{4} \Gamma_{2} \rightarrow{ }^{4} \Gamma_{4}$ transition, and the band at $640 \mathrm{~nm}$ as the ${ }^{4} \Gamma_{2} \rightarrow{ }^{4} \Gamma_{5}$ transition. After calcination a strong band at $370 \mathrm{~nm}$ is clearly seen. ${ }^{7}$ This is assigned to a chargetransfer absorption of $\mathrm{Cr}^{6+}$. The oxidation of $\mathrm{Cr}^{3+} \rightarrow \mathrm{Cr}^{6+}$ accounts for the decrease in the EPR intensity of the line in $g \approx 2.0$ (cationic sites).

Table $1 g$-Values for the crystalline zeolitic chromosilicalite obtained from fitting the experimental spectra to the powder spectra and a symmetrical line, as explained in the text; $\Delta H$ is the linewidth and $A$ is the relative amplitude

\begin{tabular}{|c|c|c|c|c|c|c|c|c|c|c|}
\hline \multirow[b]{2}{*}{ site } & \multicolumn{2}{|c|}{$g_{x}$} & \multicolumn{2}{|c|}{$g_{y}$} & \multicolumn{2}{|c|}{$g_{z}$} & \multicolumn{2}{|c|}{$\Delta H / \mathrm{kG}$} & \multicolumn{2}{|c|}{$A$ (arb. units) } \\
\hline & $300 \mathrm{~K}$ & $77 \mathrm{~K}$ & $300 \mathrm{~K}$ & $77 \mathrm{~K}$ & $300 \mathrm{~K}$ & $77 \mathrm{~K}$ & $300 \mathrm{~K}$ & $77 \mathrm{~K}$ & $300 \mathrm{~K}$ & $77 \mathrm{~K}$ \\
\hline $\mathbf{A}$ & 4.49 & 4.06 & 2.71 & 2.19 & 1.66 & 2.03 & 0.60 & 0.43 & 1.50 & 1.04 \\
\hline B & 2.00 & 2.61 & 1.67 & 1.75 & 1.66 & 1.69 & 0.66 & 0.56 & 5.23 & 3.66 \\
\hline $\mathrm{C}$ & $2.08^{a}$ & $1.88^{a}$ & & & & & 0.61 & 1.19 & 0.14 & 0.35 \\
\hline
\end{tabular}

Total $g$ value. 


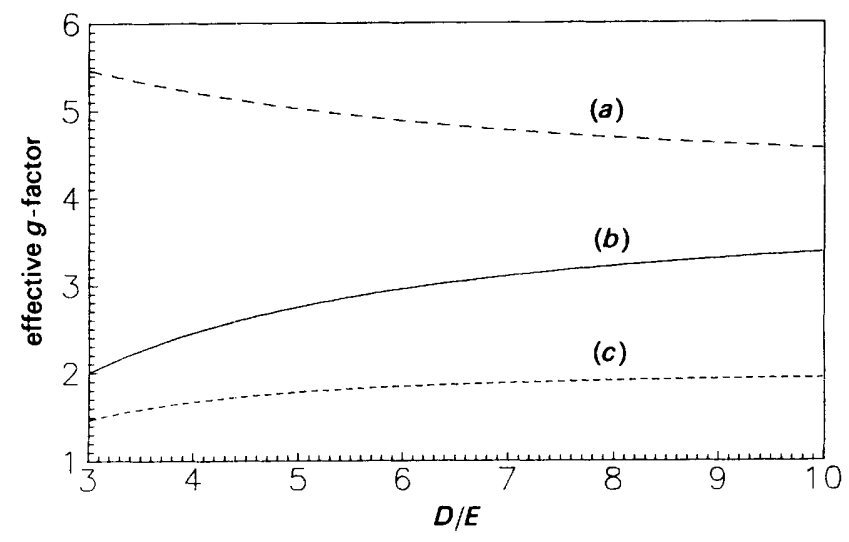

Fig. 4 Theoretical values of effective $g$-factor in the $(b) x,(a) y$ and (c) $z$ directions as a function of the ratio $E: D$

The positions of the lines of the EPR spectrum can be calculated by solving the spin Hamiltonian. ${ }^{11}$

$$
H=g \beta \boldsymbol{H} \cdot S+D\left[S_{z}^{2}-\frac{S}{3}(S+1)\right]+E\left(S_{x}^{2}-S_{y}^{2}\right)
$$

provided we can associate an effective $L=0$ value to orbital ground-state and considering tetragonal distortion from the cubic symmetry of the crystalline field. The parameters $D$ and $E$ represent the axial and rhombic distortion to the octahedron, respectively. The $\mathrm{Cr}^{3+}$ ions have the $\mathrm{d}^{3}$ electron configuration $(L=3, S=3 / 2)$ and have a larger octahedral site preference energy. ${ }^{12}$ Their interaction with the cubic crystalline field is strong and is the principal term determining the energy levels. The spin-orbit interaction and the crystalline field distortions act on the singlet orbital. The Zeeman term will then split the two Kramers doublets and the energy levels will depend on the magnetic field and $D$ and $E .{ }^{11}$ For each direction of the magnetic field several transitions may occur between the levels and we calculated their resonance field for several values of $D$ and $E$. Fig. 4 shows the expected $g$-value as a function of the ratio $D: E$ for a particular transiton. For $D: E \approx 6.5$ and $f=9.54 \mathrm{GHz}$ at room temperature, one finds a set of $g$-values in good agreement with those obtained for site A (see Table 1). For the site B we could not find any solution with these assumptions.

In order to have a better evaluation of the different contributions of each site, the samples were ion exchanged with aqueous $0.01 \mathrm{~mol} \mathrm{dm}{ }^{-3} \mathrm{HCl}$ for $24 \mathrm{~h}$ at $70-80^{\circ} \mathrm{C}$. Fig. 5 shows the EPR spectra for samples $(a)$ as synthesized and $(b)$ three times exchanged. It is clear that site B was washed away from the zeolite structure or channels.

Even though site $B$ is not easily oxidized, as would be expected for a channel species, it might be extracted by mild treatments. This leads us to believe that site B is a channel

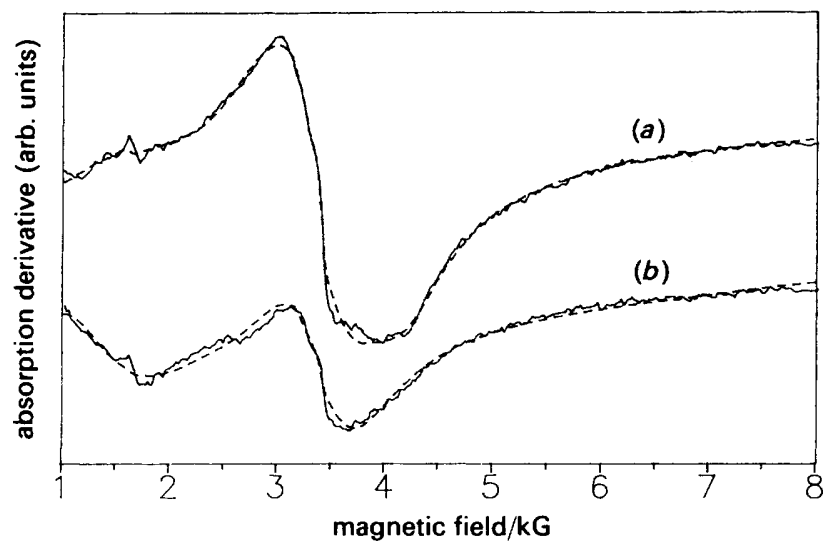

Fig. 5 EPR spectra of chromosilicalite (a) as synthesized and (b) three times ion-exchanged

species, highly distorted. That might be the reason why we were unable to find any solution for the plot of $g v s$. $D / E$ that satisfied both $\mathbf{A}$ and $\mathbf{B}$ resonances simultaneously.

The complete characterization of a series of chromosilicalites, including thermal and optical properties will be published in a forthcoming paper.

We gratefully acknowledge FAPESP, CNPq and CAPES for financial support.

\section{References}

1 C. Naccache and Y. Ben Taarit, J. Chem. Soc., Faraday Trans. 1, $1973,69,1475$.

2 V. D. Atanasova, V. A. Shvets and V. B. Kazanski, Kinet. Katal., $1977,18,1033$.

3 J. R. Pearce, D. E. Sherwood, M. B. Hall and J. H. Lunsford, J Phys. Chem., 1980, 84, 3215.

4 J. F. Hemidy, J. M. Goupil and D. Cornet, J. Chem. Phys., 1977, 74, 74.

5 A. V. Kucherov and A. A. Slinkin, Zeolites, 1987, 7, 38.

6 H. O. Pastore, E. Stein, C. U. Davanzo, E. J. S. Vichi, O. Nakamura, M. L. Baesso, E. C. da Silva and H. Vargas, J. Chem. Soc., Chem. Commun., 1990, 772.

7 J. S. T. Mambrim, E. J. S. Vichi, H. O. Pastore, C. U. Davanzo, H. Vargas, E. C. da Silva and O. Nakamura, J. Chem. Soc., Chem. Commun., 1991, 992.

8 C. P. Poole, Electron Spin Resonance, Wiley, New York, 1983, ch. 12 .

9 P. R. Bevington, Data Reduction and Error Analysis for the Physical Sciences, McGraw-Hill, New York, 1969.

10 J. C. Evans, C. R. Owen and C. C. Rowlands, J. Chem. Soc., Faraday Trans. 1, 1989, 85, 4039.

11 G. Fuxi and D. He, L. Huiming, J. Non-Cryst. Solids, 1982, 52, 135.

12 J. Wong and C. A. Angell, Glass Structure by Spectroscopy, Marcel Dekker, New York, 1976, p. 225

Paper 2/00039C; Received 6th January, 1992 